\title{
Die heutigen sympatrischen Felchenpopulationen (Coregonus spp.) des Thuner- und Bielersees und ihre Morphologie
}

\author{
Von Hans Rufli \\ Eidg. Technische Hochschule Zürich, Institut für Gewässerschutz und Wassertechnologie
}

Manuskript eingegangen am 8. August 1978

\begin{abstract}
The present sympatric whitefish populations (Coregonus spp.) of Lake Thun and Lake Biel and their morphology.

During the past 25 years the effects of eutrophication in the oligo- to mesotrophic Lake Thun and the eutrophic Lake Biel have changed the morphology and biology of the Coregonus spp., as well as the composition of the stock.

Statistical procedures and immunodiffusion revealed the existence of three Coregonus populations in Lake Thun: 5\% Kropfer, 28\% 'Albock' and 67\% Brienzlig (local names). Their gillrakers range between 20 and 32, 25 and 40, and 29 and 46, respectively (mean: 22, 31, 40). In Lake Biel two populations were distinguished: $16 \%$ Palée and $84 \%$ Bondelle with $22-35$ and $30-40$ gillrakers (mean: 30,34 ).

Changes of the spawning grounds and periods most probably have led to a bastardization of two species (Schwebalbock and Bodenalbock) to the present 'Albock' in Lake Thun.

Overlapping values of all 20 morphological characters of the populations studied render the identification of forms difficult. Only gillrakers and a few more characters show significant statistical differences between populations. The determination of Coregonus spp. of Lake Thun and Lake Biel thus often needs additional data on growth, feed, maturation, spawning ground and/or period.
\end{abstract}

\section{INHALTSVERZEICHNIS}

1. Einleitung und Problemstellung $\ldots \ldots \ldots \ldots \ldots \ldots \ldots \ldots \ldots \ldots \ldots \ldots \ldots \ldots \ldots$

2. Charakterisierung der Seen $\ldots \ldots \ldots \ldots \ldots \ldots \ldots \ldots \ldots \ldots \ldots \ldots \ldots \ldots \ldots \ldots \ldots$

3. Ermittlung der sympatrischen Populationen $\ldots \ldots \ldots \ldots \ldots \ldots \ldots \ldots \ldots \ldots \ldots \ldots \ldots \ldots . \ldots$

3.1 Die sympatrischen Populationen nach Angaben früherer Autoren $\ldots \ldots \ldots \ldots \ldots \ldots \ldots . . \ldots$

3.2 Ermittlung der heutigen sympatrischen Populationen $\ldots \ldots \ldots \ldots \ldots \ldots \ldots \ldots \ldots \ldots \ldots \ldots$

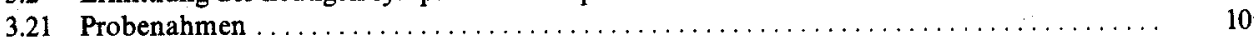

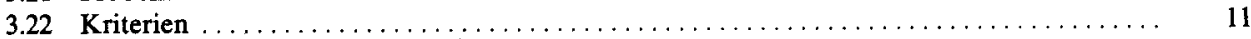

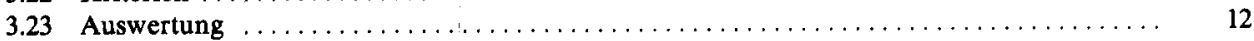

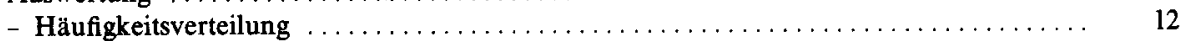

- Automatische Klassifikation $\ldots \ldots \ldots \ldots \ldots \ldots \ldots \ldots \ldots \ldots \ldots \ldots \ldots \ldots \ldots \ldots \ldots \ldots$

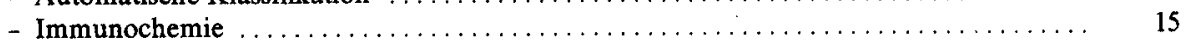

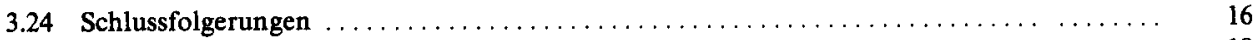

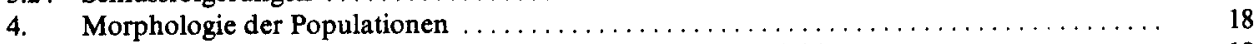

4.1 Ermittlung der Populationszugehörigkeit der einzelnen Individuen $\ldots \ldots \ldots \ldots \ldots \ldots \ldots \ldots$

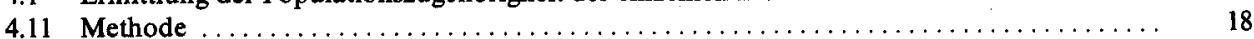

4.12 Resultate $\ldots \ldots \ldots \ldots \ldots \ldots \ldots \ldots \ldots \ldots \ldots \ldots \ldots \ldots \ldots \ldots \ldots \ldots \ldots \ldots \ldots \ldots \ldots \ldots$ 


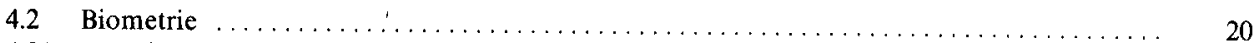

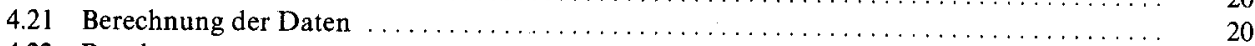

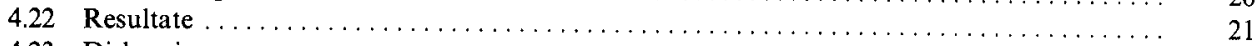

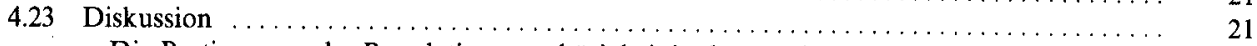

- Die Bestimmung der Populationszugehörigkeit in der Praxis .................. 21

- Vergleich mit älteren Daten . . . . . . . . . . . . . . . . . . . . . . . . . . . . . . . . . . .

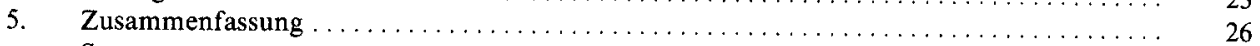

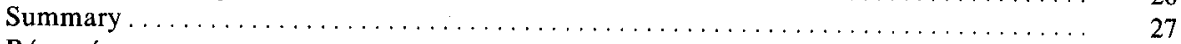

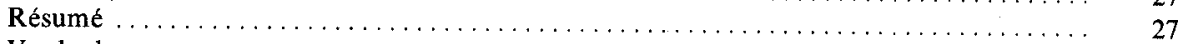

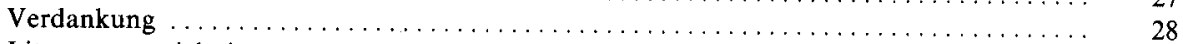

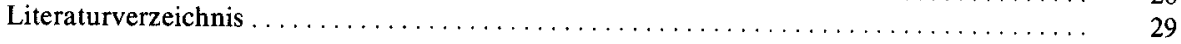

\section{Einleitung und Problemstellung}

Die Coregonen gehören in den meisten Schweizer Seen zu den wirtschaftlich wichtigsten Fischen. Durch direkte und indirekte Auswirkungen der Eutrophierung haben sich in den letzten 25 Jahren nicht nur ihre Lebensgewohnheiten, sondern auch die Bestandesgrössen und die Zusammensetzung der Arten und Rassen verändert $[17,22,29-38]$. Im Thuner- und Bielersee sind die Verhältnisse seit Steinmanns Untersuchungen in den Jahren 1940-1950 als Folge der Umweltveränderungen unklar geworden, und eine Identifizierung der Coregonen ist heute in vielen Fällen unmöglich. Die vorliegende Arbeit analysiert die Zusammensetzung des Felchenbestandes und die Unterscheidungsmerkmale der Populationen. Ihre Kenntnis ist für eine zweckmässige Bewirtschaftung der beiden Seen von Bedeutung.

Aufgrund der besonderen Schwierigkeiten der Coregonensystematik wird nicht auf die taxonomische Stellung der Populationen eingegangen.

\section{Charakterisierung der Seen}

Beim Thunersee handelt es sich um einen tiefen, oligo- bis mesotrophen Alpenrandsee (Tab. 1), während der Bielersee einen flachen, eutrophen Mittellandsee darstellt [43]. Nach dem thermischen Verhalten ist der Thunersee als subtropischer, der Bielersee als temperierter See anzusprechen [39] mit inverser Schichtung im Winter. Beide Seen werden von der Aare durchflossen, Wehre unterbinden jedoch eine Fischwanderung von See zu See.

Tabelle 1. Morphometrische und hydrologische Daten des Thuner- und Bielersees.

Table 1. Morphometrical and hydrological data of Lake Thun and Lake Biel.

\begin{tabular}{lrr}
\hline & Thunersee & Bielersee \\
\hline Meereshöhe $(\mathrm{m})$ & 560,2 & 432,1 \\
Oberfläche $\left(\mathrm{km}^{2}\right)$ & 47,9 & 40,0 \\
Länge $(\mathrm{km})$ & 18,2 & 14,8 \\
Mittlere Breite $(\mathrm{km})$ & 2,65 & 2,7 \\
Volumen $\left(\mathrm{km}^{3}\right)$ & 6,44 & 1,24 \\
Grösste Tiefe (m) & 217 & 74,6 \\
Mittlere Tiefe (m) & 135 & 31 \\
Theoretische Dauer der Wassererneuerung (Tage)* & 684 & 59 \\
\hline
\end{tabular}

* Nach Angaben des Eidg. Amtes für Energiewirtschaft. 


\title{
3. Ermittlung der sympatrischen Populationen
}

3.1 Die sympatrischen Populationen nach Angaben früherer Autoren

Die Vielfalt der Probleme der Unterscheidung der Coregonenformen und ihrer Systematik hat dazu geführt, dass die Felchenpopulationen des Thuner- und Bielersees von verschiedenen Autoren unterschiedlich bestimmt und systematisch eingeteilt wurden:

Thunersee

Fatio [10]:

- Coregonus wartmanni alpinus Bloch

- Coregonus exiguus bondella Nuesslin

- Coregonus exiguus albellus Nuesslin

- Coregonus schinzii helveticus Fatio

Wagler [62]:

- Coregonus wartmanni Bloch

- Coregonus macrophthalmus Nuesslin

- Coregonus acronius Rapp

- Coregonus fera Jurine

Trivialnamen:

«Albock»

Brienzlig

Kropfer

Balchen

«Albock»

(Blaufelchen des Bodensees)

Brienzlig

(Gangfisch des Bodensees)

Kropfer

(Kilch des Bodensees)

Balchen

(Sandfelchen des Bodensees)

Steinmann [47]:

- Coregonus lavaretus L. natio arurensis, oecotypus primigenius (Bodenalbock)

Balchen

- Coregonus lavaretus L. natio arurensis, oecotypus litoralis (Uferalbock)*

- Coregonus lavaretus L. natio arurensis, oecotypus pelagicus (Schwebfelchen)

- Coregonus lavaretus L. natio arurensis, oecotypus profundus (Tiefenalbock)

- Coregonus lavaretus L. natio arurensis, oecotypus nanus (Zwergalbock)

\author{
«Albock» \\ Kropfer \\ Brienzlig
}

*Allfällige Bezeichnung eines Uferfelchens, von dessen Existenz sich Steinmann [47] aber nicht überzeugen konnte.

Bielersee

Fatio [10]:

- Coregonus exiguus bondella Nuesslin

Trivialnamen:

- Coregonus schinzii palea Fatio

Bondelle

Palée 
- Coregonus wartmanni confusus Fatio

(Balch - Pfärrit)

Wagler [62]:

- Coregonus macrophthalmus Nuesslin

- Coregonus fera Jurine

Bondelle

Palée

Steinmann [47]:

- Coregonus L. natio jurassica, oecotypus nanus

Bondelle

- Coregonus L. natio jurassica, oecotypus primigenius*

Palée

* Unterteilt in zwei «biologische Rassen»: Palée de bord und Palée de fond.

Die Unterscheidung der Arten und Rassen basierte häufig auf Merkmalen wie Schuppenzahl, Augengrösse, Kopflänge, Umfang, Gewicht oder Abwachs [4, 12, 16, 21, 47, 62]. Nach Svaerdson [50, 51] sind von den bekannten taxonomischen Merkmalen der Coregonen aber einzig die Kiemenreusendornen umweltstabil.

\subsection{Ermittlung der heutigen sympatrischen Populationen}

Wie erwähnt, ist eine taxonomische Identifizierung der Coregonen im Thuner- und Bielersee mit Hilfe der von früheren Autoren benützten Unterscheidungsmerkmale heute in vielen Fällen unmöglich. Zudem ist die arten- und rassenmässige Zusammensetzung des Felchenbestandes unklar. Wir besitzen Anhaltspunkte dafür [42, 43], dass die zunehmende Eutrophierung Veränderungen in der Biologie und Morphologie der Fische bewirkte, die in der Folge möglicherweise auch die Zusammensetzung der Populationen beeinflusste. Es bleibt deshalb abzuklären, wie sich der Felchenbestand des Thuner- und Bielersees heute zusammensetzt.

\subsection{Probenahmen}

Vom Februar 1971 bis März 1972 wurden im Thuner- und Bielersee monatlich 20 bis 45 Fische, gesamthaft 872, aus den Fängen der Berufsfischer untersucht ${ }^{1}$ ). Die folgenden Kunstfasernetze und Maschenweiten kamen zur Anwendung:

\begin{tabular}{lllr}
\hline & $\begin{array}{l}\text { Grundnetze } \\
\text { Maschenweite }(\mathrm{mm})\end{array}$ & Tiefe $(\mathrm{m})$ & $\begin{array}{r}\text { Schwebnetze } \\
\text { Maschenweite }(\mathrm{mm})\end{array}$ \\
\hline Thunersee & $30,32,35,40,45,50$ & $8-150$ & $20^{*}, 24^{*}, 28^{*}, 35^{*}, 40,45^{*}, 50^{*}$ \\
Bielersee & $18,32,35,40$ & $1-60$ & $20^{*}, 24^{*}, 28^{*}, 35,40^{*}, 45^{*}, 50^{*}$ \\
\hline
\end{tabular}

* Kontrollfänge während März, August und Dezember 1972.

1) Das Originalmaterial befindet sich in der EAWAG/ETH, CH-6047 Kastanienbaum. Die Dissertation (Diss. Nr. 5451) ist in der ETH Zürich erhältlich. 
Im Thunersee standen Unterlagen aus den Untersuchungen von Roth [42] an rund 1100 Fischen aus den Fängen der Berufsfischer in den Jahren 1969 und 1970 zur Verfügung.

\subsection{Kriterien}

Es wurden folgende Kriterien verwendet, die sich nach den Untersuchungen von Wagler [62], Dottrens und Quartier [9], Steinmann [46, 47] und Berg und Grimaldi [3] am ehesten zur Unterscheidung der Populationen eignen (Abb. 1):

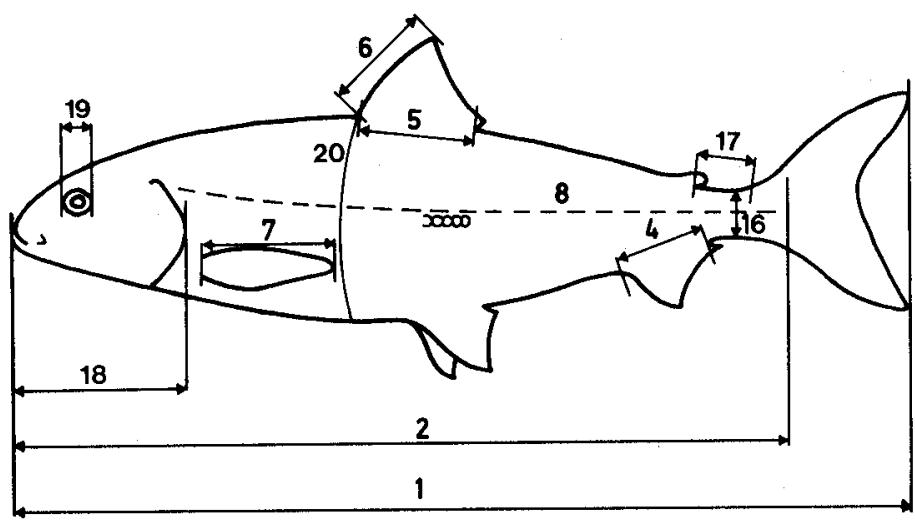

Abb. 1. Verwendete Kriterien.

Figure 1. Criterions used.

1. Totallänge (Kopfspitze bis hintere Schwanzlappen, bei normaler Spreizung).

2. Körperlänge (Kopfspitze bis letzte Schuppenquerreihe).

3. Totalgewicht.

4. Basis der Afterflosse (bei aufgestellter Afterflosse).

5. Basis der Rückenflosse (bei aufgestellter Rückenflosse).

6. Länge der Rückenflosse (bei maximal aufgestellter Rückenflosse).

7. Länge der Brustflosse (bei abgewinkelter Brustflosse).

8. Anzahl der Seitenlinienschuppen (nur perforierte Schuppen).

9. Laichausschlag (O: kein, 1: schwacher, 2: starker Ausschlag).

10. Geschlecht.

11. Reifegrad nach Nikolskii [28].

12. Fettgehalt (0: kein, 1: wenig, 2: viel, 3: sehr viel Eingeweidefett in der Leibeshöhle).

13. Gonadengewicht (Genauigkeit $\pm 0,1 \mathrm{~g}$ ).

14. Anzahl der Kiemenreusendornen auf dem ersten rechten Kiemenbogen.

15. Rückberechnete Länge des Fisches am Ende des zweiten Lebensjahres [44, 45].

Bei der Hälfte der Fische wurden zusätzlich folgende Grössen gemessen:

16. Schwanzstielhöhe (an der schmalsten Stelle). 
17. Schwanzstiellänge (vom Winkel der Fettflosse bis Ansatz des Schwanzflossenrandes).

18. Kopflänge (Spitze der geschlossenen Schnauze bis Hinterrand des Kiemendekkels).

19. Augendurchmesser (in Längsrichtung des Kopfes).

20. Umfang (mit schmalem Messband direkt vor der Rückenflosse).

\subsection{Auswertung}

\section{Häufigkeitsverteilung}

Um auf Unterschiede im Genom schliessen zu können, in unserem Fall auf verschiedene Populationen, sind morphologische Merkmale im allgemeinen ungeeignet. Sie können nämlich nicht nur vom Alter oder von der Grösse, sondern auch vom Geschlecht, von der Wachstumsrate oder dem Jahrgang der Fische beeinflusst werden [3]. Wie erwähnt, kennt man in der Coregonentaxonomie als einziges genetisches Merkmal die Anzahl der Kiemenreusendornen [48-55]. Sie werden deshalb zur Ermittlung sympatrischer Populationen mit Hilfe der Häufigkeitsverteilung herangezogen.

\section{Resultate}

Thunersee: Die polymodale Kiemenreusenverteilung in Abb. 2 deutet auf drei oder mehr Populationen hin. Die graphische Analyse des Histogramms nach Harding [13] ergibt drei Lösungsmöglichkeiten, die auch in biologischer Hinsicht denkbar wären. Den danach zu unterscheidenden hypothetischen Populationen - die vorläufig als $A, B$ und $C$ bezeichnet werden sollen, wobei $A$ und $C$ weiter in $A_{1}, A_{2}$ bzw. $C_{1}, C_{2}$ unterteilt sind - entsprechen folgende Mittelwerte der Reusendornen:

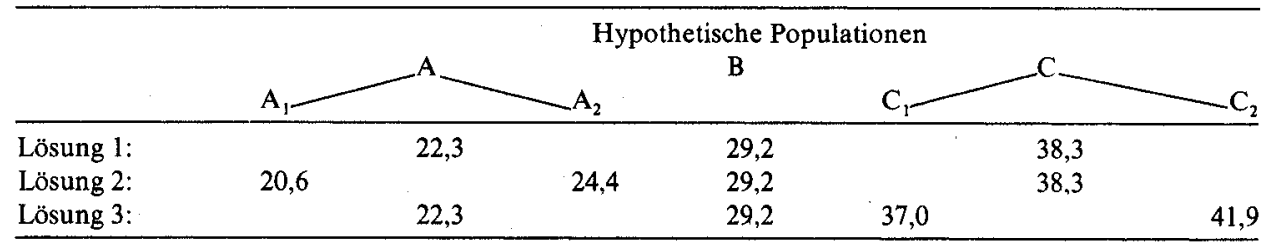

Werden die drei Lösungsmöglichkeiten mit Hilfe eines $\chi^{2}$-Tests mit der tatsächlichen Verteilung verglichen, ergeben die ersten beiden Vorschläge eine leicht bessere Anpassung $(p<0,005)$ als die dritte $(p<0,001)$.

\begin{tabular}{lrlr}
\hline & \multicolumn{1}{c}{$\chi^{2}$} & Freiheitsgrade & $p$ \\
\hline Lösung 1: & 15,2 & 4 & $<0,005$ \\
Lösung 2: & 9,1 & 1 & $<0,005$ \\
Lösung 3: & 14,5 & 1 & $<0,001$ \\
\hline
\end{tabular}

$\mathrm{Ob}$ sich eine weitere Unterteilung von $\mathrm{A}$ oder $\mathrm{C}$ in zwei Populationen rechtfertigt, soll in der weiteren Untersuchung überprüft werden. 
Lokalisiert man innerhalb der Verteilung die von den Fischern unterschiedenen Formen (die oft falsch angesprochen werden), lässt sich einzig der Kropfer einer unserer hypothetischen Populationen zuordnen: Population A (nur durch wenige Exemplare vertreten). Die übrigen Formen sind auf die Populationen B und C verstreut.

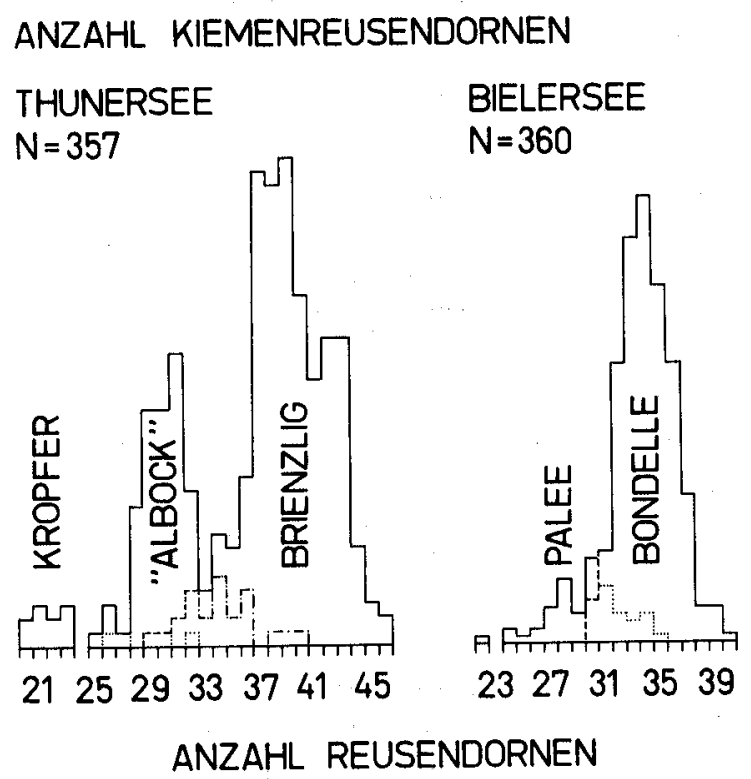

Abb. 2. Häufigkeitsverteilung der Anzahl der Kiemenreusendornen der Thuner- und Bielerseefelchen. Figure 2. Pooled frequency distribution of gillrakers of Coregonus spp. of Lake Thun and Lake Biel.

Bielersee: Im Bielersee scheinen nach dem Histogramm zwei Populationen vorhanden zu sein (Abb.2), was durch die graphische Analyse bestätigt wird. Es ergeben sich folgende zwei Möglichkeiten:

\begin{tabular}{llllll} 
& \multicolumn{2}{l}{ Hypothetische Populationen } & $\chi^{2}$ & Freiheitsgrade & p \\
& A & B & & & $<0,25$ \\
\hline Lösung 1: & 28,6 & 33,6 & 14,8 & 11 & $<0,1$ \\
Lösung 2: & 25,7 & 33,4 & 17,9 & 10 & $<$ \\
\hline
\end{tabular}

Die erste Lösung der tatsächlichen Verteilung ist besser angepasst $(p<0,25$ gegenüber $\mathrm{p}<0,1)$.

Bei den Tieren mit niederen Kiemenreusenzahlen handelt es sich ausschliesslich um die vom Fischer als Palée bezeichnete Form, die heute sehr selten geworden ist. Durch den kleinen Gipfel im Histogramm bei 27-28 Reusendornen wird sie schwach angedeutet. Die übrigen Tiere sind, nach den Fischern, Vertreter der Bondelle. 
Automatische Klassifikation

Die Tatsache, dass die Fischer den Kropfer und den Palée identifizieren können (zumindest während eines gewissen Zeitabschnitts, z. B. Laichzeit), zeigt, dass sich diese Formen offenbar nicht nur in ihrer Kiemenreusenzahl, sondern auch in andern Merkmalen unterscheiden. Mit den Verfahren der automatischen Klassifikation soll geprüft werden, welche Populationen unter gleichzeitiger Berücksichtigung mehrerer Merkmale in den beiden Seen zu unterscheiden sind.

\section{Methode}

Bei dem agglomerativen Verfahren nach Ward [63] bildet in der Ausgangssituation jeder Fisch mit seinen Merkmalen eine separate Gruppe. Im Agglomerationsschritt werden diejenigen zwei Gruppen zusammengefasst, deren Gruppenmittelpunkte am nächsten beieinanderliegen. Diese Kombination der zwei ähnlichsten Gruppen wird so lange wiederholt, bis alle Objekte in einer einzigen Gruppe vereinigt sind.

\section{Eindeutigkeit der Resultate}

Das Verfahren ergibt eine Anzahl Klassierungen (mit abnehmender Zahl Gruppen) und die dazugehörige Streuung. Damit ist das Resultat nicht eindeutig bestimmt. In Kombination mit andern Verfahren lässt sich die subjektive Beeinflussung jedoch auf ein Minimum reduzieren.

\section{Kriterien}

Durch voneinander unabhängige Klassifikationen, je mit Fischen gleichen Alters, Geschlechts und Fangmonats, soll eine Klassierung nach diesen Faktoren vermieden werden. Als Eingangsdaten der automatischen Klassifikation werden die unkorrelierten Werte der Hauptkomponentenanalyse folgender Kriterien benützt:

- Totallänge/Gewicht.

- Relative Länge der Afterflossenbasis.

- Relative Rückenflossenlänge.

- Relative Länge der Rückenflossenbasis.

- Relative Brustflossenlänge.

- Länge/Basis der Rückenflosse.

- Rückenflossenbasis/Afterflossenbasis.

- Anzahl Kiemenreusendornen.

- Kiemenreusendornen/Seitenlinienschuppen.

- Anzahl Seitenlinienschuppen.

- Relative Schwanzstielhöhe.

- Relative Schwanzstiellänge.

- Schwanzstielhöhe/Schwanzstiellänge.

- Länge am Ende des zweiten Lebensjahres [44, 45].

- Reifegrad nach Nikolskii [28].

- Relatives Gonadengewicht.

- Laichausschlag.

- Fettgehalt.

- Relative Kopflänge. 
- Relativer Umfang.

- Brustflossenlänge/Kopflänge.

- Augengrösse/Kopflänge.

\section{Resultate}

Thunersee: Drei voneinander unabhängige Klassifikationen (mit verschiedenen Gruppen von Fischen) erbringen ein einheitliches Ergebnis: eine Auftrennung in Klassen, die mit den hypothetischen Populationen A, B und C übereinstimmen.

Aus der Analyse geht zudem hervor, dass innerhalb der Populationen B und C unabhängig von der Kiemenreusenzahl grössere Differenzen bestehen als innerhalb der Population A.

Bielersee: Die beiden Klassifikationen der Bielerseefelchen ergeben eine klare Auftrennung in die hypothetischen Populationen A und B.

\section{Immunochemie}

Qualitative proteinanalytische Methoden wie Elektrophorese, Ouchterlony-Immunodiffusion und Immunoelektrophorese gestatten einen direkten Nachweis von einzelnen Proteinen und damit Genen oder Gensequenzen. Mit ElektrophoreseUntersuchungen an Tieren des Genus Coregonus gelang es einerseits, Fischpopulationen zu trennen [14, 15, 24, 58-61], anderseits verunmöglichten starke Variationen in den Elektropherogrammen eine Charakterisierung der untersuchten Tiere [5, 40, $41,56,57]$. In den wenigen bisherigen immunologischen Versuchen konnten Populationen getrennt werden $[2,11]$.

Mit Hilfe der Ouchterlony-Immunodiffusion an Gonadenextrakten der Thunerseefelchen soll festgestellt werden, inwieweit sich die aus der Häufigkeitsverteilung und automatischen Klassifikation hervorgegangenen Populationen immunochemisch unterscheiden lassen.

\section{Material und Methode}

Untersuchte Fische: Zur Untersuchung gelangten 18 laichreife Tiere der Populationen A, B und C, die an demselben Tag (Ausnahme: Population A) auf den Laichplätzen gefangen wurden.

Herstellung von Gonadenextrakten: $1 \mathrm{mg}$ der Ovarien (Reifegrad nach Nikolskii [28]: 4-5) wurde zu gleichen Teilen mit Quarzsand zerrieben und 0,2 $\mathrm{ml}$ Phosphatpuffer ( $1 / 15$ M, pH 7,2 nach Sørensen) zugegeben. Das Extrakt wurde nach 10 Minuten Zentrifugation (Christ-Zeta-20-Zentrifuge) bei $10000 \mathrm{U} . / \mathrm{min}$ erhalten.

Herstellung von Gonadenantiserum: Durch wöchentliche intramuskuläre Injektionen von $500 \mu$ l Gonadenextrakten mit $500 \mu$ l Freund's adjuvant wurden Meerschweinchen während 5 Wochen immunisiert. In der sechsten Woche wurde das Blut durch Herzpunktation entnommen. Die Seren lagerten anschliessend für 8 Monate bei $-20^{\circ} \mathrm{C}$.

Doppeldiffusion: Die Diffusion wurde während 48 Stunden bei $1-4^{\circ} \mathrm{C}(7,5 \times 4,5-\mathrm{cm}$ Platten) in Agarose durchgeführt. Die zentrale Vertiefung enthielt $100 \mu$ l Antiserum, die peripheren $50 \mu \mathrm{l}$ der Gonadenextrakte. Nach dem Waschen in physiologischer Kochsalzlösung (72 Std.) wurden die Präzipitate mit Thiazinrot T gefärbt. 


\section{Resultate}

Die Ouchterlony-Diffusion ergibt klare Differenzen durch sich kreuzende Präzipitate (und damit genetische Unterschiede) zwischen $A$ und $C$ sowie $B$ und $C$ (Abb.3). Zudem bestehen Unterschiede zwischen $A$ und $C$ sowie A und B in der Anzahl der Fällungslinien.
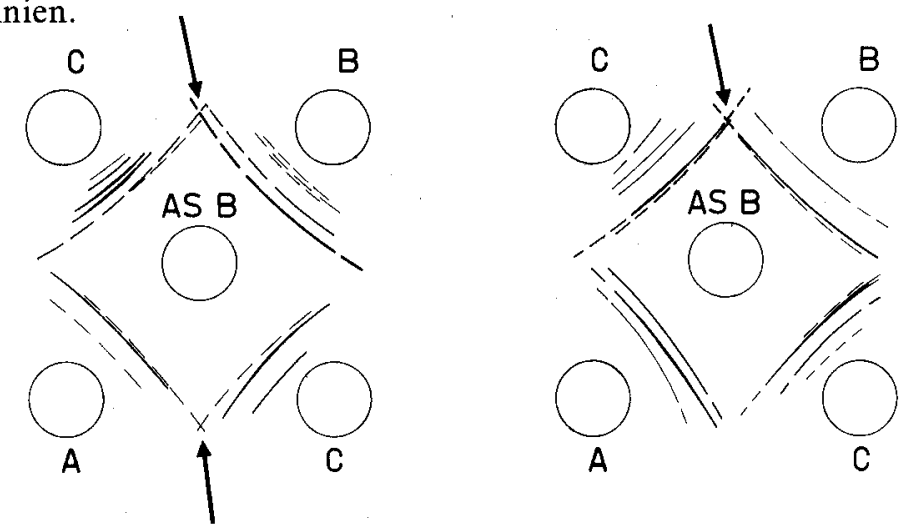

Abb. 3. Ouchterlony-Diffusion mit Gonadenextrakten der hypothetischen Populationen A. B. C im Thunersee und dem Antiserum der Population B (ASB). Pfeile: Sich kreuzende Präzipitate, die auf genetische Unterschiede hinweisen.

Figure 3. Double diffusion using extracts of gonads of the hypothetical populations A, B, C of Lake Thun, and the antiserum of population B (ASB). Arrows: crossing precipitates pointing out genetical differences.

\subsection{Schlussfolgerungen}

Die wahrscheinlichsten Lösungen der Analyse der Kiemenreusenverteilung entsprechen sowohl den Ergebnissen der automatischen Klassifikation als auch jenen der Immunodiffusion für die Thunerseefelchen. Die durch die Diskontinuität im Kiemenreusendiagramm bei 24 und 41 Kiemenreusen abgetrennten Populationen sind deshalb höchst wahrscheinlich identisch mit A bzw. C. Dafür sprechen auch die folgenden Gründe:

- Aus der automatischen Klassifikation geht hervor, dass innerhalb der Population A die geringsten Differenzen bestehen.

- Die Diskontinuität bei 24 Kiemenreusendornen könnte auch durch die zu geringe Zahl an Tieren der Population A hervorgerufen sein.

- Es bestehen keine statistisch gesicherten Differenzen zwischen den Populationen $C_{1}$ und $C_{2}$ bezüglich der Laichzeit (Tiere mit Kiemenreusenzahlen über 36 laichen von August bis Dezember).

- Fische mit 33 bis 46 Kiemenreusendornen wurden auf denselben Laichplätzen gefangen.

Wir schliessen deshalb auf drei sympatrische Populationen im Thunersee mit den folgenden Anteilen an der Gesamtfelchenpopulation:

Anteil an Gesamtpopulation (\%) 
In Anlehnung an die von früheren Autoren unterschiedenen Formen und den von ihnen verwendeten Bezeichnungen nennen wir die Populationen nach der Zahl der Kiemenreusendornen:

\begin{tabular}{|c|c|c|c|c|c|}
\hline \multicolumn{3}{|c|}{ Nach vorliegender Arbeit } & \multicolumn{3}{|c|}{ Nach Steinmann [47] } \\
\hline \multirow[b]{2}{*}{ Bezeichnung } & \multicolumn{2}{|c|}{ Kiemendornen } & \multicolumn{2}{|c|}{ Kiemendornen } & \multirow[b]{2}{*}{ Bezeichnung } \\
\hline & Bereich & Mittel & Bereich & Mittel & \\
\hline A: Kropfer & $20-32$ & 22 & $13-25$ & & Kropfer (Tiefenalbock) \\
\hline \multirow[t]{2}{*}{ B: «Albock» } & $25-40$ & 31 & $20 \div 37$ & 27 & Balchen (Bodenalbock) \\
\hline & & & $22-36$ & 34 & Albock (Schwebalbock) \\
\hline C: Brienzlig & $29-46$ & 40 & $32-42$ & & Brienzlig (Zwergalbock) \\
\hline
\end{tabular}

Wie bereits erwähnt, wird die Population A auch vom Fischer als Kropfer (Tiefenalbock) identifiziert und nicht etwa als Balchen (Bodenalbock) oder Albock (Schwebalbock), wie man nach dem Bereich der Kiemenreusendornen allein auch schliessen könnte [42]. Der heutige Kropfer ist mit der von Steinmann beschriebenen Form des Tiefenalbocks (Kropfer), aber zusätzlich aufgrund der Laichzeit, des Laichplatzes, der Nahrung und der Aufenthaltstiefe [44] sowie des grösseren relativen Augendurchmessers und der längeren relativen Brustflosse identisch. Die Population B entspricht dem Balchen des Fischers und z.T. dem Albock (Schwebalbock). Die Population $\mathrm{C}$ wird hingegen vom Fischer nebst Brienzlig häufig als Albock angesprochen (ursprünglich ein Kleinfelchen, unterscheidet er sich heute in der Grösse nur mehr wenig vom früheren Balchen und Albock [44, 45]). Die Population $C$ ist nebst den Kiemenreusen auch nach dem Wachstum, z.T. nach der Laichzeit und dem Laichplatz und nach der Mehrzahl der morphologischen Merkmale (in ihrer relativen Grösse zueinander), soweit Vergleichswerte bestehen, dem Brienzlig von Steinmann gleichzusetzen.

Um die heutige Form des Albocks vom früheren Schlag des Albocks (Schwebalbock) zu unterscheiden, soll sie in der Folge als «Albock» gekennzeichnet werden. Analog resultieren im Bielersee zwei sympatrische Populationen:

Anteil an Gesamtpopulation (\%)

Hypothetische Population A 16

Hypothetische Population B

die den von den Fischern und früheren Autoren als:

\begin{tabular}{|c|c|c|c|c|c|}
\hline & \multicolumn{2}{|c|}{$\begin{array}{l}\text { Nach vorlie- } \\
\text { gender Arbeit } \\
\text { Kiemendornen }\end{array}$} & \multicolumn{2}{|c|}{$\begin{array}{l}\text { Nach Dottrens und } \\
\text { Quartier [9] } \\
\text { Kiemendornen }\end{array}$} & \multirow{2}{*}{$\begin{array}{r}\text { Nach } \\
\text { Steinmann }[47] \\
\text { Kiemendornen } \\
\text { Bereich } \\
\end{array}$} \\
\hline & Bereich & Mittel & Bereich & Mittel & \\
\hline A: Palée & $22-35$ & 30 & $21-35$ & 27 & $19-32$ \\
\hline B: Bondelle & $30-40$ & 34 & $26-41$ & 34 & $26-35$ \\
\hline
\end{tabular}

bezeichneten Populationen entsprechen. 


\section{Morphologie der Populationen}

\subsection{Ermittlung der Populationszugehörigkeit der einzelnen Individuen}

Die Ermittlung der Populationszugehörigkeit von einzelnen Individuen anhand morphologischer Unterschiede allein ist, wie erwähnt, in vielen Fällen nicht mit Sicherheit möglich. Es empfiehlt sich deshalb, weitere Kriterien heranzuziehen, wie Laichgewohnheiten, Wachstum, Alter und Länge beim Eintritt der Geschlechtsreife. Mit statistischen Verfahren, die eine vorgeschlagene Gruppierung (nach den Reusenzahlen) unter gleichzeitiger Berücksichtigung mehrerer Merkmale einschätzen und aufgrund gewisser Kriterien verbessern, lassen sich einzelne Individuen anschliessend einer Gruppe zuordnen.

\subsection{Methode}

Nach dem Verfahren von Demirmen [6] wird die vorgeschlagene Gruppierung durch die Summe über alle Gruppenstreuungen (Kompaktheit) geschätzt. Durch Umgruppierung entsprechender Individuen nach dem Algorithmus des «nächsten Nachbarn» (analog der «k-means»-Methode von McQueen [27]) wird die Summe reduziert und die Klassierung damit in bezug auf ihre Kompaktheit verbessert. Die neu erhaltene Einteilung wird in der Folge auf gleiche Weise geschätzt. Dieser Algorithmus wird so lange fortgesetzt, bis keine Verbesserung der Kompaktheit mehr möglich ist.

Die Berechnung basiert auf den orthonormalisierten Daten (Ưbergewichtung einzelner Merkmale wird verhindert) folgender Zahlenwerte:

- Anzahl Reusendornen auf dem ersten rechten Kiemenbogen.

- Gewicht.

- Relative Afterflossenbasis.

- Relative Rückenflossenbasis.

- Relative Rückenflossenlänge.

- Relative Brustflossenlänge.

- Rückenflossenbasis/Afterflossenbasis.

- Rückenflossenlänge/Rückenflossenbasis.

- Seitenlinienschuppenzahl.

- Reifegrad nach Nikolskii [28].

- Laichausschlag.

- Relatives Gonadengewicht.

- Fettgehalt.

- Länge am Ende des ersten Lebensjahres [44, 45].

- Anzahl Reusendornen/Seitenlinienschuppen.

\subsection{Resultate}

Die aus dem Verfahren resultierenden Kiemenreusenverteilungen der einzelnen Populationen sind in Abb. 4 dargestellt. 


\title{
ANZAHL KIEMENREUSENDORNEN
}

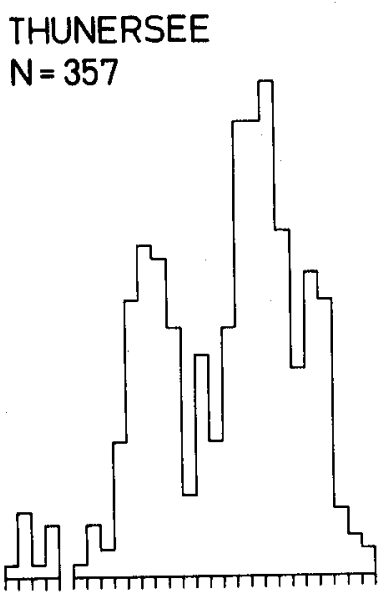

\section{BIELERSEE \\ $N=358$}
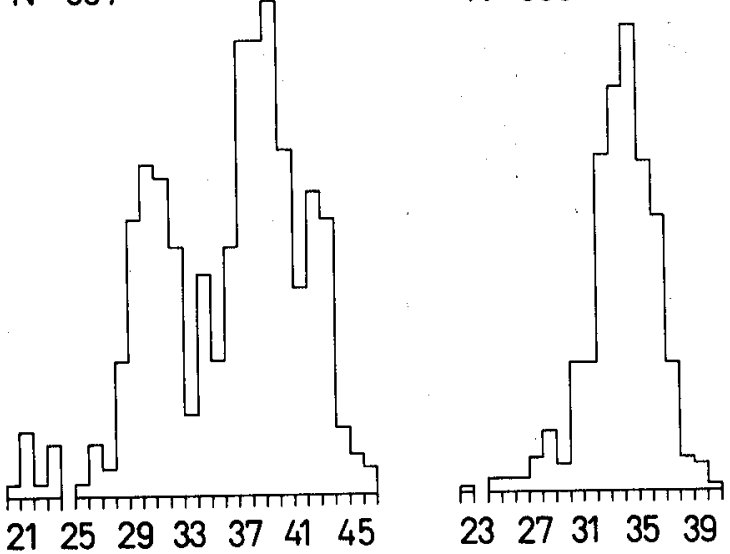

\begin{abstract}
Abb.4. Häufigkeitsverteilung der Anzahl der Kiemenreusendornen der unterschiedenen Thuner- und Bielerseefelchenpopulationen.

Figure 4. Frequency distribution of the number of gillrakers of the separated Coregonus populations in Lake Thun und Lake Biel.
\end{abstract}

\section{ANZAHL REUSENDORNEN}

Tabelle 2. Statistisch gesicherte Differenzen zwischen den Populationen (Totallängen der Fische im Thunersee: $28-32 \mathrm{~cm}$, im Bielersee: $28-30 \mathrm{~cm}$ ).

Table 2. Significant differences between populations (total length of specimens of Lake Thun: $28-32 \mathrm{~cm}$, of Lake Biel: $28-30 \mathrm{~cm}$ ).

\begin{tabular}{|c|c|c|c|c|}
\hline & $\begin{array}{l}\text { Thunersee } \\
\text { "Albock»/ } \\
\text { Brienzlig (p) }\end{array}$ & $\begin{array}{l}\text { «Albock»/ } \\
\text { Kropfer }(p)\end{array}$ & $\begin{array}{l}\text { Brienzlig/ } \\
\text { Kropfer (p) }\end{array}$ & $\begin{array}{r}\text { Bielersee } \\
\text { Bondelle/ } \\
\text { Palée (p) } \\
\end{array}$ \\
\hline Anzahl Kiemenreusendornen & $<0,001$ & $<0,001$ & $<0,001$ & $<0,001$ \\
\hline Anzahl Seitenlinienschuppen & & & $<0,05$ & \\
\hline Relative Afterflossenbasis & $<0,01$ & & & $<0,001$ \\
\hline Relative Rückenflossenbasis & $<0,001$ & & $<0,005$ & \\
\hline Relative Rückenflossenlänge & $<0,001$ & & $<0,001$ & \\
\hline Relative Brustflossenlänge & $<0,001$ & $<0,001$ & $<0,001$ & \\
\hline Brustflossenlänge/Rückenflossenlänge & $<0,001$ & & & \\
\hline Rückenflossenlänge/Rückenflossenbasis & & & & \\
\hline Rückenflossenbasis/Afterflossenbasis & $<0,001$ & & & \\
\hline Relative Schwanzstiellänge & & & $<0,01$ & \\
\hline Relative Schwanzstielhöhe & $<0,001$ & $<0,005$ & & \\
\hline Schwanzstielhöhe/Schwanzstiellänge & $<0,001$ & & & \\
\hline Relative Kopflänge & & $<0,001$ & $<0,001$ & \\
\hline Relative Augengrösse & $<0,05$ & $<0,001$ & $<0,001$ & \\
\hline Augendurchmesser/Kopflänge & $<0,005$ & $<0,005$ & $<0,005$ & \\
\hline Relativer Umfang & & & & \\
\hline Totallänge/Gewicht & & & & \\
\hline Gewicht & & & & \\
\hline Kiemenreusendornen/Seitenlinienschuppen & $<0,001$ & $<0,001$ & $<0,001$ & $<0,001$ \\
\hline N-Faktor* $(9)$ & $<0,001$ & $<0,001$ & $<0,001$ & $<0,001$ \\
\hline
\end{tabular}

* $\mathrm{N}=\frac{\text { Rückenflossenbasis }}{\text { Afterflossenbasis }}+$ Seitenlinienschuppen $-\frac{\text { Brustflossenlänge }}{\text { Rückenflossenbasis }} 3 \times$ Kiemenreusendornen. 
Tabelle 3. Werte der morphologischen Unterscheidungsmerkmale der Felchenpopulation des Thunersees. Table 3. Values of the morphological characteristics of Coregonus populations of Lake Thun.

\begin{tabular}{|c|c|c|c|c|c|c|c|c|c|c|c|c|}
\hline \multirow[t]{2}{*}{ Merkmal } & \multicolumn{4}{|c|}{ Kropfer } & \multicolumn{4}{|c|}{ «Albock» } & \multicolumn{4}{|c|}{ Brienzlig } \\
\hline & TL & $\mathbf{M}$ & ST & $\mathbf{N}$ & $\mathrm{TL}$ & $\mathbf{M}$ & ST & $\mathrm{N}$ & TL & $\mathrm{M}$ & ST & $\mathrm{N}$ \\
\hline \multirow[t]{6}{*}{ Anzahl Kiemenreusen } & 0 & 23,5 & 3,7 & 18 & 0 & 30,8 & 2,7 & 98 & 0 & 39,0 & 3,0 & 239 \\
\hline & 260 & 25,5 & 4,9 & 6 & 260 & 30,5 & 2,7 & 15 & 220 & 42,3 & 0,8 & 6 \\
\hline & 300 & 22,3 & 2,1 & 12 & 300 & 31,3 & 3,6 & 34 & 260 & 40,6 & 2,9 & 40 \\
\hline & & & & & 340 & 30,3 & 2,0 & 32 & 300 & 39,6 & 3,0 & 79 \\
\hline & & & & & 380 & 30,6 & 1,6 & 14 & 340 & 38,0 & 2,8 & 104 \\
\hline & & & & & 420 & 30,6 & 1,1 & 5 & 380 & 38,0 & 3,0 & 19 \\
\hline Länge der Brustflosse & 0 & 157,9 & 16,3 & 18 & 0 & 136,1 & 9,1 & 97 & 0 & 136,5 & 10,0 & 236 \\
\hline \multirow[t]{5}{*}{ Totallänge } & 260 & 157,3 & 21,8 & 6 & 260 & 133.8 & 11,9 & 15 & 220 & 140,3 & 13,3 & 6 \\
\hline & 300 & 160,2 & 12,8 & 11 & 300 & 133,0 & 7,6 & 33 & 260 & 141,5 & 9,9 & 38 \\
\hline & & & & & 340 & 135,3 & 7,4 & 30 & 300 & 138,4 & 10,8 & 74 \\
\hline & & & & & 380 & 143,8 & 7,9 & 13 & 340 & 133,1 & 8,3 & 102 \\
\hline & & & & & 420 & 145,0 & 4,3 & 5 & 380 & 135,6 & 7,7 & 16 \\
\hline Augengrösse & 0 & 37,5 & 2,9 & 11 & 0 & 31,4 & 3,1 & 45 & 0 & 31,2 & 3,3 & 59 \\
\hline \multirow[t]{4}{*}{ Totallänge } & 260 & 38,8 & 3,9 & 5 & 260 & 34,4 & 3,9 & 7 & 260 & 33,4 & 3,1 & 20 \\
\hline & 300 & 36,3 & 1,2 & 6 & 300 & 32,3 & 3,0 & 14 & 300 & 30,5 & 2,4 & 20 \\
\hline & & & & & 340 & 30,5 & 1,9 & 11 & 340 & 29,1 & 2,4 & 16 \\
\hline & & & & & 380 & 29,6 & 2,6 & 7 & & & & \\
\hline Augengrösse & 0 & 223,3 & 18,6 & 11 & 0 & 198,4 & 18,4 & 46 & 0 & 199,1 & 21,8 & 60 \\
\hline \multirow[t]{5}{*}{ Kopflänge } & 260 & 223,0 & 29,0 & 5 & 260 & 212,9 & 24,1 & 7 & 260 & 211,3 & 25,7 & 20 \\
\hline & 300 & 223,5 & 4,8 & 6 & 300 & 205,5 & 16,8 & 14 & 300 & 193,8 & 14,9 & 20 \\
\hline & & & & & 340 & 193,8 & 12,7 & 11 & 340 & 188,1 & 16,0 & 16 \\
\hline & & & & & 380 & 191,0 & 14,1 & 7 & & & & \\
\hline & & & & & 420 & 189,0 & 13,0 & 5 & & & & \\
\hline Anzahl Kiemenreusen & 0 & 276,7 & 40,8 & 18 & 0 & 359,2 & 32,5 & 98 & 0 & 445,9 & 47,2 & 239 \\
\hline \multirow{5}{*}{$\overline{\text { Anz. Seitenlinienschuppen }}$} & 260 & 292,7 & 56,3 & 6 & 260 & 356,6 & 34,1 & 15 & 220 & 502,2 & 19,6 & 6 \\
\hline & 300 & 264,2 & 27,3 & 11 & 300 & 365,3 & 41,7 & 33 & 260 & 486,0 & 41,8 & 40 \\
\hline & & & & & 340 & 355,0 & 26,1 & 30 & 300 & 459,3 & 42,3 & 74 \\
\hline & & & & & 380 & 354,5 & 21,0 & 13 & 340 & 420,5 & 37,1 & 102 \\
\hline & & & & & 420 & 355,0 & 23,2 & 5 & 380 & 419,7 & 26,8 & 16 \\
\hline
\end{tabular}

TL: Totallänge, M: Mittelwert, ST: Standardabweichung, N: Anzahl Individuen, 0: alle Grössenklassen, 220: Grössenklasse 200-239 mm, 260: Grössenklasse 240-279 mm, 300: Grössenklasse 280-319 mm, 340: Grössenklasse 320-359 mm.

\subsection{Biometrie}

\subsection{Berechnung der Daten}

Um Vergleichswerte zu den Untersuchungen anderer Autoren zu erhalten, wurden im wesentlichen diejenigen Merkmale und Merkmalskombinationen berechnet (Mittelwerte und Standardabweichungen; die Merkmale und ihre Verhältnisse zueinander erwiesen sich als normalverteilt), die auch in den Arbeiten von Wagler [62], Steinmann [46, 47], Dottrens und Quartier [9] und Berg und Grimaldi [3] verwendet wurden (Tab.2). Für die Grössenklassen $28-32 \mathrm{~cm}$ im Thunersee und 28-30 cm im Bielersee (bei 28-32-cm-Klassen unterscheiden sich Palée und Bondelle in ihrer mittleren Länge $\mathrm{p}<0,01$ ) wurde zudem geprüft, ob statistisch gesicherte Differenzen zwischen den Populationen bestehen. 
Tabelle 4. Werte der morphologischen Unterscheidungsmerkmale der Felchenpopulationen des Bielersees.

Table 4. Values of the morphological characteristics of Coregonus populations of Lake Biel.

TL: Totallänge, M: Mittelwert, ST: Standardabweichung, N: Anzahl Individuen, 0: alle Grössenklassen, 220: Grössenklasse 200-239 mm, 260: Grössenklasse 240-279 mm, 300: Grössenklasse 280-319 mm, 340: Grössenklasse 320-359 mm.

\begin{tabular}{|c|c|c|c|c|c|c|c|c|}
\hline \multirow[t]{2}{*}{ Merkmal } & \multicolumn{4}{|c|}{ Palée } & \multicolumn{4}{|c|}{ Bondelle } \\
\hline & TL & $\mathbf{M}$ & ST & $\mathrm{N}$ & TL & $\mathbf{M}$ & ST & $\mathrm{N}$ \\
\hline \multirow[t]{5}{*}{ Anzahl Kiemenreusen } & 0 & 29,6 & 2,7 & 56 & 0 & 34,1 & 1,9 & 304 \\
\hline & 260 & 28,9 & 1,7 & 7 & 260 & 33,5 & 1,7 & 61 \\
\hline & 300 & 30,3 & 2,6 & 40 & 300 & 34,3 & 1,9 & 231 \\
\hline & 340 & 27,2 & 1,7 & 4 & 340 & 35,6 & 2,7 & 5 \\
\hline & 380 & 25,5 & 1,9 & 4 & & & & \\
\hline Anzahl Kiemenreusen & 0 & 338,5 & 36,1 & 56 & 0 & 396,7 & 26,9 & 304 \\
\hline \multirow{4}{*}{$\overline{\text { AnzahlSeitenlinienschuppen }}$} & 260 & 333,1 & 30,0 & 7 & 260 & 391,6 & 26,8 & 61 \\
\hline & 300 & 347,0 & 33,8 & 40 & 300 & 397,7 & 26,5 & 231 \\
\hline & 340 & 307,2 & 21,2 & 4 & 340 & 402,0 & 40,0 & 5 \\
\hline & 380 & 288,0 & 28,6 & 4 & & & & \\
\hline Basis der Afterflosse & 0 & 97,3 & 8,1 & 55 & 0 & 100,3 & 10,0 & 301 \\
\hline \multirow{4}{*}{ Totallänge } & 260 & 100,7 & 8,5 & 7 & 260 & 102,0 & 10,5 & 61 \\
\hline & 300 & 97,3 & 7,6 & 40 & 300 & 99,7 & 6,5 & 231 \\
\hline & 340 & 99,0 & 12,8 & 4 & 340 & 102,8 & 6,9 & 5 \\
\hline & 380 & 89,0 & 3,0 & 3 & & & & \\
\hline
\end{tabular}

\subsection{Resultate}

In den Ergebnissen kommt die für die Coregonen bekannte grosse Variationsbreite der morphologischen Merkmale zum Ausdruck. Auffallend ist aber die Überschneidung der Extremwerte der verschiedenen Formen, selbst bei Merkmalen oder Merkmalskombinationen, in denen sich die Populationen am stärksten unterscheiden (Tab.3, 4). Einzig die Kiemenreusenzahlen und das Verhältnis derselben zur Zahl der Seitenlinienschuppen sowie der N-Faktor nach Dottrens und Quartier [9] weisen statistisch gesicherte Differenzen zwischen sämtlichen Populationen der beiden Seen auf (Tab.2). Die Felchenformen des Thunersees unterscheiden sich ausserdem bezüglich der Brustflossenlänge, der Augengrösse und der Kopflänge sowie deren Verhältnis zueinander. Palée und Bondelle besitzen unterschiedliche Längen der Afterflossenbasis (Tab.2).

Morphologische Differenzen bestehen vor allem zwischen Brienzlig und «Albock» bzw. Brienzlig und Kropfer, während sich die beiden Felchenformen des Bielersees äusserlich überhaupt kaum zu unterscheiden scheinen (Abb.5-9).

\subsection{Diskussion}

\section{Die Bestimmung der Populationszugehörigkeit in der Praxis}

Die Populationszugehörigkeit der Felchen des Thuner- und Bielersees lässt sich anhand der statistisch gesicherten, morphologischen Differenzierungsmerkmale 


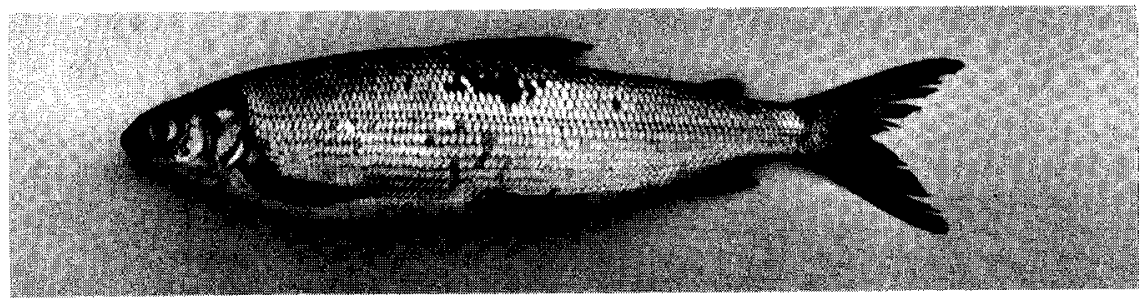

Abb.5. Kropfer des Thunersees. Totallänge $280 \mathrm{~mm}$, Gewicht $170 \mathrm{~g}$, Alter $4^{+}$. Figure 5. Kropfer of Lake Thun. Total length $280 \mathrm{~mm}$, weight $170 \mathrm{~g}$, age $4^{+}$.

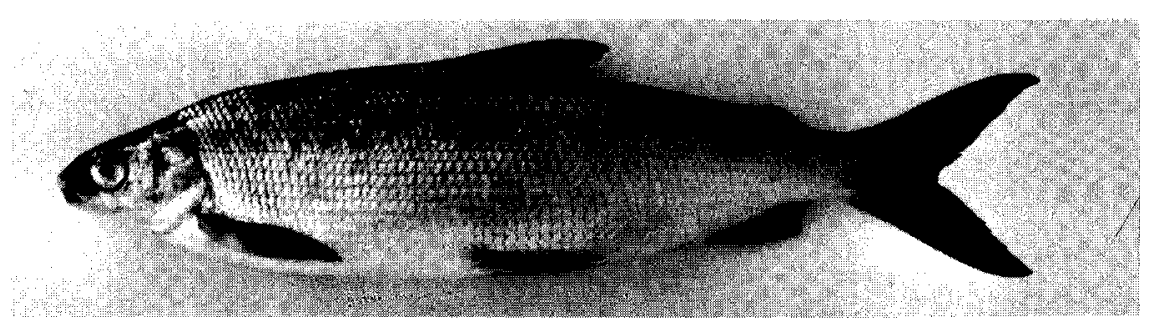

Abb. 6. «Albock» des Thunersees. Totallänge $350 \mathrm{~mm}$, Gewicht $225 \mathrm{~g}$, Alter $3^{+}$. Figure 6. 'Albock' of Lake Thun. Total length $350 \mathrm{~mm}$, weight $225 \mathrm{~g}$, age $3^{+}$.

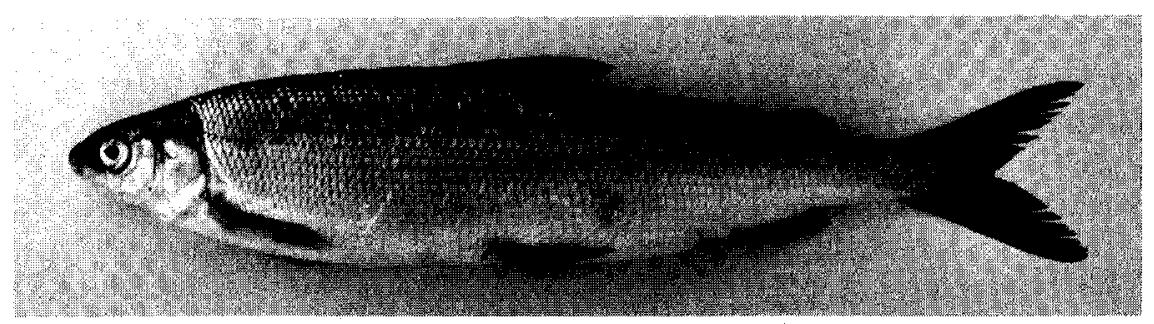

Abb. 7. Brienzlig des Thunersees. Totallänge $323 \mathrm{~mm}$, Gewicht $260 \mathrm{~g}$, Alter $4^{+}$.

Figure 7. Brienzlig of Lake Thun. Total length $323 \mathrm{~mm}$, weight $260 \mathrm{~g}$, age $4^{+}$.

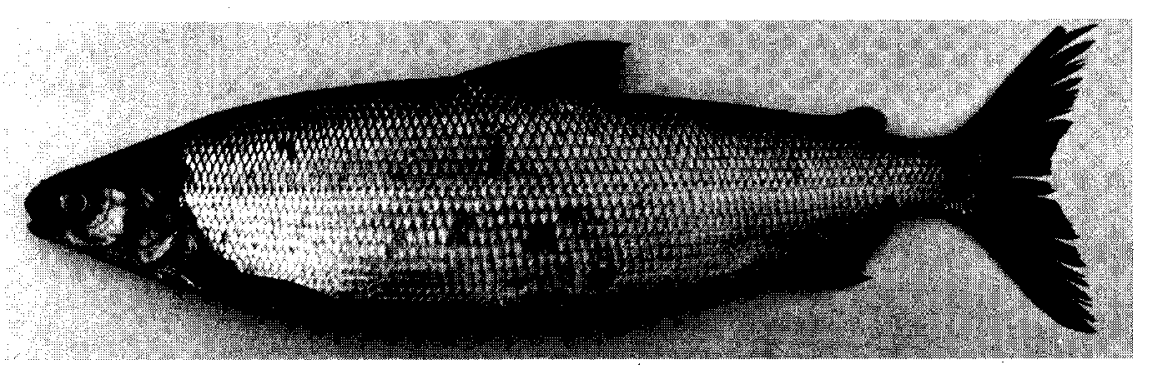

Abb. 8. Palée des Bielersees. Totallänge $430 \mathrm{~mm}$, Gewicht $780 \mathrm{~g}$, Alter $4^{+}$.

Figure 8. Palée of Lake Biel. Total length $430 \mathrm{~mm}$, weight $780 \mathrm{~g}$, age $4^{+}$. 


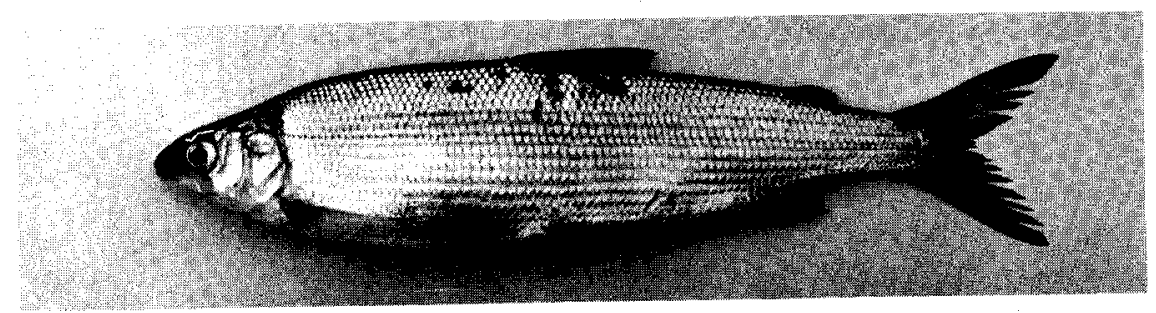

Abb.9. Bondelle des Bielersees. Totallänge $300 \mathrm{~mm}$, Gewicht $225 \mathrm{~g}$, Alter $3^{+}$.

Figure 9. Bondelle of Lake Biel. Total length $300 \mathrm{~mm}$, weight $225 \mathrm{~g}$, age $3^{+}$.

allein nicht immer eindeutig bestimmen. Jede zusätzliche Information über Wachstum, Nahrung, Eintritt der Geschlechtsreife kann deshalb wertvoll sein, insbesondere sind es Angaben über Laichplätze und Laichzeiten der betreffenden Fische. Um eine allfällige Bestimmung der Thuner- und Bielerseefelchen zu erleichtern, sind in den Tabellen 5 und 6 sämtliche Differenzierungsmerkmale zusammengestellt, welche aus der vorliegenden Arbeit sowie aus Untersuchungen über die Ernährung und das Wachstum [44, 45] als auch über die Fortpflanzung [44] hervorgingen.

\section{Vergleich mit älteren Daten}

Eine Gegenüberstellung der Kiemenreusenzahlen der heutigen Populationen im Thunersee (als wichtigstem Merkmal der Coregonentaxonomie) mit den Ergebnissen von Steinmann [47] ergibt deutliche Abweichungen.

Obwohl beim Kropfer nur 18 Individuen zur Verfügung stehen, dürfen wir annehmen, dass sich die Anzahl der Reusendornen in den letzten 25 Jahren um mindestens vier Einheiten erhöht hat (Bereich der Reusendornen bei Steinmann [47]: 13-25, eigene Untersuchungen: 20-32, Mittel 22). Auffallend ist vor allem, dass Tiere mit extrem geringen Zahlen sowohl im Material der Untersuchungen von Roth [42] als auch im eigenen vollständig fehlen.

Falls es sich dabei nicht um eine scheinbare Verschiebung handelt, hervorgerufen durch zahlenmässig zu geringe Proben, kann sie in diesem Umfang nicht allein durch veränderte Umweltbedingungen verursacht worden sein. Nach bisherigen Erfahrungen haben solche Änderungen 2,4 Einheiten nicht überschritten [18, 20,21, 26]. Grössere Abweichungen, wahrscheinlich als Folge natürlicher oder/und künstlicher Selektion, sind hingegen von Svaerdson [48-55] und Berg und Grimaldi [3] festgestellt worden. Letztere konnten an der Bondella des Lago Maggiore zudem eine Abhängigkeit von der Wachstumsrate während der ersten beiden Lebensjahre nachweisen. Das Wachstum sämtlicher Thuner- und Bielerseefelchen hat sich in den letzten 25 Jahren tatsächlich stark verbessert [44, 45]. Anderseits sind auch genetische Verschiebungen denkbar. Es ist bekannt, dass zahlenmässig geschwächte Populationen - wie der Kropfer - eher zur Hybridisierung neigen [25]. Die überlappenden Laichzeiten und Laichplätze würden eine Vermischung insbesondere von Kropfer und dem ursprünglichen Schwebalbock sowie von Kropfer und Brienzlig gestatten. In beiden Fällen führte eine Hybridisierung zu einer Erhöhung der 
Tabelle 5. Unterscheidungsmerkmale der Felchenpopulationen des Thunersees.

Table 5. Characteristics of Coregonus populations of Lake Thun.

\begin{tabular}{|c|c|c|c|}
\hline & Kropfer & «Albock» & Brienzlig \\
\hline $\begin{array}{l}\text { Morphologie } \\
\text { (Mittelwerte für Totallängen } 28-32 \mathrm{~cm} \text { ) }\end{array}$ & & & \\
\hline $\begin{array}{l}\text { Reusendornen } \\
\text { Brustflossenlänge/Totallänge } \\
\text { Augendurchmesser/Totallänge } \\
\text { Augendurchmesser/Kopflänge } \\
\text { Reusendornen/Seitenlinienschuppen }\end{array}$ & $\begin{array}{r}22 \\
160 \\
363 \\
224 \\
264\end{array}$ & $\begin{array}{r}31 \\
133 \\
323 \\
206 \\
365\end{array}$ & $\begin{array}{r}40 \\
138 \\
305 \\
194 \\
459\end{array}$ \\
\hline Nahrung & $\begin{array}{l}\text { Vorwiegend Boden- } \\
\text { nahrung, häufig } \\
\text { Chironomiden }\end{array}$ & \multicolumn{2}{|c|}{$\begin{array}{l}\text { Vorwiegend Zooplankton, häufig } \\
\text { Daphnia. Im Oktober/November } \\
\text { v.a. Brienzlig, auch Chironomiden }\end{array}$} \\
\hline $\begin{array}{l}\text { Wachstum } \\
\text { (mittlere Jahresendlänge in } \mathrm{cm} \text { ) }\end{array}$ & & & . \\
\hline $\begin{array}{l}\text { 1. Altersjahr } \\
\text { 2. Altersjahr } \\
\text { 3. Altersjahr } \\
\text { 4. Altersjahr } \\
\text { 5. Altersjahr }\end{array}$ & $\begin{array}{l}14 \\
24 \\
27 \\
29\end{array}$ & $\begin{array}{l}15 \\
27 \\
33 \\
37 \\
38\end{array}$ & $\begin{array}{l}10 \\
22 \\
28 \\
30 \\
31\end{array}$ \\
\hline Eintritt der Geschlechtsreife & & & \\
\hline $\begin{array}{l}\text { Alter } \\
\text { Totallänge }\end{array}$ & $\begin{aligned} & 2-3 \\
> & 26\end{aligned}$ & $\begin{aligned} & 1-2 \\
> & 25\end{aligned}$ & $>20$ \\
\hline Laichzeit & Ende Juli-Dezember & Mitte Dezember & ugust-Dezember \\
\hline Laichplatz & $\begin{array}{l}40-150 \mathrm{~m} \text { Tiefe, } \\
\text { vorwiegend } \\
\text { Bachdeltas }\end{array}$ & $\begin{array}{l}\text { Ufer, ca. 0-30 m Tiefe } \\
\text { vorwic }\end{array}$ & $\begin{array}{l}20-80 \mathrm{~m} \text { Tiefe, } \\
\text { gend Bachdeltas, } \\
\text { z.T. im Schweb, } \\
\text { am Ufer oder } \\
>80 \mathrm{~m} \text { Tiefe }\end{array}$ \\
\hline
\end{tabular}

Kiemenreusenzahlen. Eine stärkere Vermischung des Kropfers mit andern Populationen müsste aber darüber hinaus auch Veränderungen eines Teils der übrigen Merkmale mit sich bringen. Nach Andriasheva [1] sind Hybridisierungen oft auch von einer Vergrösserung des Nahrungsspektrums begleitet. Wie erwähnt, unterscheidet sich der heutige Kropfer allerdings sehr wenig von der von Steinmann [47] beschriebenen Form. Nach Rufli $[44,45]$ sind sie bezüglich Laichzeit, Laichplatz, Nahrung und Aufenthaltstiefe identisch. Ebenso besitzt der Kropfer noch heute längere Brustflossen sowie grössere Augen und einen längeren Kopf als "Albock» und Brienzlig (soweit Vergleichswerte bestehen, sind dies die einzigen Merkmale, in denen Unterschiede zu erkennen sind). Deshalb vermuten wir, dass sich der Kropfer nicht in starkem Masse mit andern Populationen vermischt hat und die Erhöhung der Kiemenreusenzahlen eher auf die grössere Wachstumsrate und/oder Selektion zurückzuführen ist.

Steinmann [47] unterschied im Bereich von etwa 20-37 Kiemenreusendornen zwei Populationen: den Bodenalbock (Balchen) mit 20-37, im Mittel 27, und den 
Tabelle 6. Unterscheidungsmerkmale der Felchenpopulationen des Bielersees.

Table 6. Characteristics of Coregonus populations of Lake Biel.

\begin{tabular}{llr}
\hline & Palée & Bondelle \\
\hline $\begin{array}{l}\text { Morphologie } \\
\text { (Mittelwerte für Totallängen } 28-30 \mathrm{~cm} \text { ) }\end{array}$ & & 30 \\
Reusendornen & 347 & 34 \\
Reusendornen/Seitenlinienschuppen & 973 & 398 \\
Afterflossenbasis/Totallänge & & 997 \\
& Unreife und Verlaichte & Wenig Felcheneier \\
Nahrung & im Dezember, z. T. im Januar & im Dezember und Januar \\
& vorwiegend Felcheneier &
\end{tabular}

Wachstum

(mittlere Jahresendlänge in $\mathrm{cm}$ )

$\begin{array}{ll}\text { 1. Altersjahr } & 18 \\ \text { 2. Altersjahr } & 27 \\ \text { 3. Altersjahr } & 29 \\ \text { 4. Altersjahr } & 31 \\ \text { 5. Altersjahr } & 35\end{array}$

Eintritt der Geschlechtsreife

Alter

Totallänge

Schwebalbock (Albock) mit 22-36, im Mittel 34 Reusendornen. Die damaligen Hauptunterscheidungsmerkmale, nämlich Laichzeiten und Laichplätze, haben sich seither stark verändert. Die Laichzeit des Schwebalbocks hat sich seit dem 19. Jahrhundert (September) immer mehr gegen das Jahresende hin verschoben, bis sie schliesslich mit der Balchenlaichzeit im Dezember zusammenfiel. Nach Steinmann deckte sich die Laichfangperiode der Schwebalböcke bereits 1950 praktisch mit jener der Bodenalböcke. Die Laichplätze der beiden Schläge überlappten sich wahrscheinlich an ihren Grenzen ebenso. Während meiner Untersuchungen konnten auf den ursprünglichen Laichplätzen der Schwebalböcke nur zwei Tiere gefangen werden, die in bezug auf Kiemenreusenzahl, Laichplatz und Laichzeit einem Schwebalbock entsprachen. Bei den übrigen Tieren mit demselben Fangort handelte es sich ausschliesslich um den Brienzlig. Diese Ergebnisse lassen vermuten, dass es während der letzten 25 Jahre zum Zusammenbruch der Isolationsbarrieren und in der Folge zu einer Introgression (im Sinne von Mayr [25]) gekommen ist. Nach den Kiemenreusenzahlen und Laichgewohnheiten (identisch mit jenen des Bodenalbocks) der heutigen "Alböcke» zu beurteilen, stellen diese einen Hybriden aus Schwebalbock und Bodenalbock dar. Ähnliche Vermischungsprozesse sind aus dem Bodensee [17, 38], dem Starnberger See [19] und vor allem aus kleineren Seen bekannt $[7,8,23]$. 
Beim Brienzlig stehen den früheren Werten von 28 bis 42 Reusendornen heute 29 bis 46 gegenüber. Die Zahl der Kiemenreusen hat sich demnach um etwa vier Einheiten erhöht. Als Ursachen kommen ebenfalls Modifikationen, natürliche Selektion und die von Berg und Grimaldi festgestellte Abhängigkeit von der Wachstumsrate in Frage. Infolge des drastisch verbesserten Wachstums werden demnach heute beim Auszählen der Kiemenreusen kleine Dornen mitgezählt, die bei den früheren Zwergalböcken nicht sichtbar waren. Auszuschliessen ist eine stärkere Vermischung mit andern Formen, denn der Brienzlig besitzt die höchsten Reusenzahlen. Eine solche hätte daher eine Verminderung der Kiemenreusenzahlen mit sich gebracht.

Im Bielersee stimmen die Werte der Bondelle mit den von Dottrens und Quartier [9] gefundenen Zahlen der Bondelle aus dem Neuenburgersee überein. Beim Palée hingegen liegt der Mittelwert um drei Einheiten höher. Es ist durchaus möglich, dass auch diese in starkem Rückgang begriffene Form zur Introgression neigt. Teilweise kommt es in den Brutanstalten zur künstlichen Bastardierung. Wie beim Brienzlig können Veränderungen aber auch hier durch natürliche und/oder künstliche Selektion, Modifikation oder durch das verbesserte Wachstum [3] verursacht werden.

Dass unter diesen Umständen viele der Werte der übrigen Merkmale und Merkmalskombinationen nicht mehr den früheren entsprechen, ist nicht erstaunlich. Svaerdson [51, 53-55] hat durch Umsetzen («transplantations») von Populationen in andere Gewässer wiederholt den Einfluss der Umwelt auf die bekannten taxonomischen Coregonenmerkmale nachweisen können. Dieselben Veränderungen zeigten sich an der aus dem Neuenburgersee stammenden Bondella des Lago Maggiore [3]. Die Autoren schreiben dazu, dass sich die Bondella des Lago Maggiore von der Bondelle des Neuenburgersees heute stärker unterscheidet als vom Lavarello des Lago Maggiore. Ihre Untersuchungen ergaben, dass neben der Grösse des Fisches die Wachstumsrate, der Jahrgang, das Alter, das Geschlecht und das Fanggebiet einen bestimmenden Einfluss auf morphologische Merkmale ausüben.

\section{Zusammenfassung}

Die Auswirkungen der Eutrophierung haben die Morphologie und die Biologie der Coregonen im Thuner- (oligo- bis mesotroph) und Bielersee (eutroph) verändert. Die vorliegende Arbeit hatte zum Ziel, Angaben über die arten- oder rassenmässige Zusammensetzung sowie über die Charakteristiken der Populationen als Basis für eine zweckmässige Bewirtschaftung zu liefern.

Aufgrund der Ergebnisse der Reusendornenverteilung, der multivariaten Analyse biometrischer und biologischer Merkmale sowie der Immunodiffusion von Gonadenextrakten sind im Thunersee drei sympatrische Felchenformen zu unterscheiden: $5 \%$ Kropfer, 28\% «Albock» und 67\% Brienzlig (Trivialnamen). Die Bereiche der Reusendornen betragen 20-32, 25-40 und 29-46 (Mittel: 22, 31, 40) (Abb.4). Im Bielersee ergeben sich zwei Populationen Palée und Bondelle mit 16 und $84 \%$ Anteil am Felchenbestand und 22-35 bzw. 30-40 Reusendornen (Mittel: 30, 34) (Abb.4). Die von Steinmann beschriebenen Schläge Balchen (Bodenalbock) und Albock (Schwebalbock) des Thunersees können heute nicht mehr voneinander differenziert 
werden. Aufgrund der Änderungen der Laichzeiten und Laichplätze kam es während der letzten 25 Jahre vermutlich zum Zusammenbruch der Isolationsbarrieren und in der Folge zu einer Introgression. Nach den Kiemenreusendornen und Laichgewohnheiten handelt es sich beim heutigen «Albock» um einen Hybriden aus ursprünglichem Albock (Schwebalbock) und Balchen (Bodenalbock). Durch Selektion, verbessertes Wachstum und/oder genetische Verschiebungen hat sich die Anzahl Reusendornen des Kropfers, Brienzligs und der Bondelle erhöht.

Von sämtlichen 20 untersuchten morphologischen Merkmalen überschneiden sich die Extremwerte der verschiedenen Formen (Tab.3, 4). Nur die Reusendornen, deren Kombination mit der Zahl der Seitenlinienschuppen und der N-Faktor weisen statistisch gesicherte Differenzen zwischen sämtlichen Populationen auf (Tab.2). Die Formen im Thunersee unterscheiden sich ausserdem bezüglich der Brustflossenlänge, der Augengrösse und der Kopflänge sowie deren Verhältnis zueinander. Palée und Bondelle besitzen unterschiedliche Längen der Afterflossenbasis (Tab. 2). Morphologische Unterschiede bestehen vor allem zwischen «Albock» und Brienzlig sowie Kropfer und Brienzlig (Abb.5-9). Für eine Bestimmung der Felchen des Thuner- und Bielersees sind z.T. zusätzliche Angaben über Wachstum, Ernährung, Eintritt der Geschlechtsreife und/oder Laichzeit und Laichplatz notwendig (Tab. 5, 6).

\section{SUMMARY}

The effects of eutrophication in the oligo- to mesotrophic Lake Thun and the eutrophic Lake Biel have changed the morphology and biology of the Coregonus spp. The objective of this paper is to obtain data on composition and population characteristics for a purposeful management of the Coregonus stock.

By means of analyses of gillraker distributions, of biometrical and biological characters, and of immunodiffusion of gonad extracts, the present study reveals the existence of three populations in Lake Thun: 5\% Kropfer, 28\% 'Albock' and 67\% Brienzlig (local names). Their gillrakers range between 20 and 32, 25 and 40 and 29 and 46, respectively (mean: 22, 31, 40) (fig.4). In Lake Biel two stocks are distinguished: $16 \%$ Palée and $84 \%$ Bondelle with $22-35$ and $30-40$ gillrakers (mean: 30,34 ) (fig.4).

The forms Balchen (Bodenalbock) and Albock (Schwebalbock), as described by Steinmann, cannot be distinguished anymore. Due to changes of spawning grounds and periods during the past 25 years, the isolation barriers most probably collapsed and led to an introgression. According to the number of gillrakers and the spawning behaviour, the present 'Albock' resulted from a bastardization of the former Albock (Schwebalbock) and Balchen (Bodenalbock). Selection, improved growth rate and/or genetical shiftings have increased the number of gillrakers of Kropfer, Brienzlig and Bondelle

The extreme values of all 20 morphological characters of the various populations studied overlap and render the identification of the forms difficult (tables 3,4 ). Only gillrakers, the ratio between gillrakers and the number of lateral line scales, and the $\mathrm{N}$-factor show significant differences between all populations (table 2). Moreover the forms in Lake Thun differ in regard to the length of the pectoral fin, the longitudinal iris diameter and the head length. Palée and Bondelle show different lengths of the anal fin base (table 2). Morphological differences exist mainly between 'Albock' and Brienzlig, and Kropfer and Brienzlig (fig.5-9). The determination of Coregonus spp. of Lake Thun and Lake Biel often needs additional data on growth, feed, maturation, spawning ground and/or period (tables 5,6 ).

\section{RÉSUMÉ}

Les répercussions d'eutrophication dans le lac de Thoune (oligo- jusqu'à mesotrophe) et le lac de Bienne (eutrophe) ont changé la morphologie et la biologie des Corégones. Le présent travail a pour but d'obtenir des données sur la composition et les caractéristiques de ces populations et de fournir une base adéquate concernant l'économie piscicole. 
Les analyses des distributions de branchiospines, des caractères biométriques et biologiques et de l'immunodiffusion des extraits de gonade démontrent l'existence de trois populations dans le lac de Thoune: $5 \%$ de Kropfer, $28 \%$ d' «Albock» et $67 \%$ de Brienzlig (noms locaux). Leurs branchiospines varient entre 20 et 32,25 et 40 et 29 et 46 (moyennes: $22,31,40$ ) (fig. 4). Le lac de Bienne contient deux populations: $16 \%$ de Palée et $84 \%$ de Bondelle avec 22-35, respectivement $30-40$ branchiospines (moyennes: 30,34 ) (fig. 4).

Les formes Balchen (Bodenalbock) et Albock (Schwebalbock) du lac de Thoune, décrites par Steinmann, ne peuvent plus être différenciées. Dû au changement des frayères et des saisons du frai pendant les 25 dernières années, les barrières d'isolation se sont probablement effondrées, ce qui mena à une introgression. Selon le nombre des branchiospines et des habitudes de frai l' "Albock» d'aujourd'hui est le résultat d'une hybridation de l'ancien Albock (Schwebalbock) et du Balchen (Bodenalbock). Par sélection, amélioration de la croissance et/ou changement génétique le nombre des branchiospines des Kropfer, Brienzlig et Bondelle a augmenté.

Les valeurs extrêmes de tous les 20 caractères morphologiques des différentes populations étudiées chevauchent et rendent difficile l'identification des formes (tabl.3, 4). Parmi toutes les populations, seuls les branchiospines, leur rapport avec le nombre des écailles de la ligne latérale ainsi que l'indice $\mathrm{N}$ ont montré des différences significantes (tabl.2). En plus, les formes que l'on trouve dans le lac de Thoune se distinguent par la longueur des nageoires pectorales, par le diamètre de l'œil et la longueur de la tête ainsi que par la relation entre l'œil et la tête. La longueur de la base des nageoirs anales diffère chez Palée et Bondelle (tabl.2). Des différences morphologiques existent surtout entre «Albock» et Brienzlig et entre Kropfer et Brienzlig (fig. 5-9). La détermination des Corégones des lacs de Thoune et de Bienne demandent souvent des informations supplémentaires sur la croissance, la nourriture, la maturation sexuelle, frayère et/ou période du frai $($ tabl.5,6).

\section{VERDANKUNG}

Herrn Prof. Dr. W. Stumm, Direktor der EAWAG, und Herrn Prof. Dr. O. Jaag, alt Direktor, möchte ich danken, dass sie die Durchführung dieser Arbeit ermöglichten. Besonderen Dank möchte ich dem Leiter der Abt. Fischereiwissenschaften an der EAWAG, Herrn Dr. W. Geiger, aussprechen, der die Arbeit anregte und mit seinen wertvollen Ratschlägen und seiner Hilfe unterstützte. Danken möchte ich auch Herrn Privatdozent Dr. R. Peduzzi für persönliche Hilfe und Einführung in das Gebiet der Elektrophorese und Immunologie.

Für das stetige Interesse und die persönliche Unterstïtzung der Untersuchungen danke ich Herrn Prof. Dr. H. Roth, Kantonaler Fischereiinspektor des Kantons Bern, herzlich. Besonders dankbar bin ich den Fischereiaufsehern, Herren W. Röthlisberger, H. Fahrni, H. Greber, H. Roth, A. Wittwer, H. Perrod, H. Ballif, und den Berufsfischern des Brienzer-, Thuner- und Bielersees, vor allem den Herren H. Kaufmann, R. und F. Thomann, A. und E. Meyer, W. Sieber, H. Tschanz, H. Tschan, K. Furer, A. Martin, H. Pilloud, A. Grimm, H. Dubler und H.P. Dasen. Die vielen Probenahmen waren nur mit der Hilfe dieser Herren möglich. Zeitweise halfen mir auch Frl. C. Kühn und Studienkollegen, denen ich ebenfalls meinen besten Dank aussprechen möchte. Schliesslich danke ich Herrn Dr. A. Keller für anregende Diskussionen und Hinweise sowie für die Durchsicht des Manuskriptes.

Die Arbeit wurde mit Unterstützung der «Stiftung der Wirtschaft zur Förderung des Gewässerschutzes in der Schweiz" durchgeführt.

\section{LITERATURVERZEICHNIS}

1 Andriasheva, M.A.: Some results obtained by the hybridization of Cyprinids. Proceedings of the World Symposium on Warmwater pond fish culture, Rome 18-25/5/1966, F.A.O. Fish. Rep. 44/4, 205-214 (1966).

2 Bargetzi, J.P.: Applications de méthodes d'analyses biochimique à un problème taxonomique: les Corégones du lac de Neuchâtel. Schweiz. Z. Hydrol. 22, 641-759 (1960).

3 Berg, A., und Grimaldi, E.: Biologia delle due forme di Coregone (Coregonus sp.) del Lago Maggiore. Memorie Ist. ital. Idrobiol. 18, 25-196 (1956).

4 Berg, L.S.: The freshwater fishes of U.S.S.R. and the surrounding countries (Russisch), Teil I, 4. Aufl. Moskau 1948. 
5 Booke, H.E.: Variability of the electrophoretic patterns of blood serum proteins in a whitefish, Coregonus clupeaformis, population. M.S. thesis, Michigan State University, 1962.

6 Demirmen, F.: Multivariate procedures and Fortran IV program for evaluation and improvement of classifications, 50 p. Stanford University of Kansas, Lawrence 1969.

7 Dottrens, E.: Acclimatation et hybridation de Corégones. Revue suisse Zool. 62, 101-118 (1955).

8 Dottrens, E.: Systématique des Corégones de l'Europe occidentale, basée sur une étude biométrique. Revue suisse Zool. 66, 1-66 (1959).

9 Dottrens, E., und Quartier, A.: Les Corégones du Lac de Neuchâtel. Etude biométrique. Revue suisse Zool. 56, 689-730 (1949).

10 Fatio, V.: Faune des Vertébrés de la Suisse, Bd. V, 2.Teil. Genf, Basel 1890.

11 Fenderson, O.C.: Evidence of subpopulations of lake whitefish Coregonus clupeaformis, involving a dwarfed form. Trans. Am. Fish. Soc. 93/1, 77-94 (1964).

12 Freidenfelt, T.: Untersuchungen über die Coregonen des Wenersees. Int. Revue ges. Hydrobiol. Hydrogr. 30, 49-163 (1934).

13 Harding, J.P.: The use of probability paper for the graphical analysis of polymodal frequency distributions. J. Mar. Biol. 28, 141-153 (1949).

14 Hodgins, H.O., Ames, E.E., und Utter, F. M.: Variants of lactate dehydrogenase isozymes in sera of sockeye salmon (Oncorhynchus nerka). J. Fish. Res. Bd Can. 26, 15-19 (1969).

15 Hodgins, H.O., und Utter, F.M.: Lactate dehydrogenase polymorphism of sockeye salmon (Oncorhynchus nerka). Cons. Perma. Int. Explor. Mer., Rapp. Proc.-Verb. Réun. 161, 100-101 (1971).

16 Hubbs, L., und Lagler, K.: Fishes of Great lakes region. The Cranbook Press, Michigan 1947.

17 Karbe, L.: Die Auswirkung der künstlichen Eutrophierung des Bodensees auf das Artgefüge seiner Coregonenpopulationen. Mitt. Hamburg. Zool. Mus. Inst. Kosswig-Festschrift, S.83-90 (1964).

18 Kliever, E.V.: Gillraker variation and diet in lake whitefish Coregonus clupeaformis in Northern Manitoba. In: Lindsey, C.C., und Woods, C.S. (eds.): Biology of Coregonid fishes, 560 p. University of Manitoba Press, Winnipeg 1970.

19 Kölbing, A.: Der Starnberger See und die seinem Trophiezustand angemessene Bewirtschaftungsweise des Coregonenbestandes. Veröff. zool. Staatssamml. München 17, 1-108 (1974).

20 Koelz, W.: Coregonid fishes of Great Lakes. Bull. U.S. Bur. Fish. 43/2, 297-643 (1929).

21 Koelz, W.: The Coregonid fishes of northeastern America. Pap. Mich. Acad. Sci., Arts Lett. 13, 303432 (1931).

22 Kriegsmann, F.: Der Wechsel in der Vergesellschaftung der Fischarten des Ober- und Untersees und die Veränderungen des See-Reagierens. Arch. Hydrobiol. 22, 397-408 (1955).

23 Lampert, W.: Untersuchungen zur Biologie und Populationsdynamik der Coregonen im Schluchsee. Arch. Hydrobiol., suppl. 38, 237-314 (1971).

24 Lindsey, C.C., Clayton, J.W., und Franzin, W. G.: Zoogeographic problems and protein variation in the Coregonus clupeaformis whitefish species complex. In: Lindsey, C.C., und Woods, C.S. (eds.): Biology of Coregonid fishes, 560 p. University of Manitoba Press, Winnipeg 1970.

25 Mayr, E.: Artbegriff und Evolution, 617 p. Paul-Parey-Verlag, Hamburg und Berlin 1967.

26 McCart, P., und Andersen, B.: Plasticity of gillraker number and length in Oncorhynchus nerka. J. Fish. Res. Bd Can. 24/9, 1999-2002 (1967).

27 McQueen, J.: Some methods for classification of slight alterations in numerical technique. Syst. Zool. 14, 196-213 (1966).

28 Nikolskii, G.V.: The ecology of fishes, 352 p. Academic Press, London, New York 1963.

29 Nümann, W.: Vorläufiger Bericht über das stark veränderte Wachstum der Blaufelchen im Bodensee und Versuch einer Fangprognose für das Jahr 1958. Allg. Fisch.-Ztg. 83, 88-91 (1958).

30 Nümann, W.: Das Wachstum der Blaufelchen und seine Berechnung bei Erfassung aller Individuen der einzelnen Jahrgänge in Abhängigkeit von der Stärke des Jahrganges, den jährlich variierenden Aussenfaktoren, der Eutrophierung und den regionalen Unterschieden des Sees. Arch. Fisch.-Wiss. 10, 5-20 (1959).

31 Nümann, W.: Das Problem der Ertragssteigerung und Überfischung in der Blaufelchenfischerei am Bodensee. Z. Fisch. Hilfswiss. 10 (N.F.), 241-252 (1961).

32 Nümann, W.: Schnelleres Wachstum, grössere Fangerträge, jüngere Jahrgänge und Frühreife bei den Bodenseeblaufelchen als Folge der Düngung des Sees durch Abwässer. Allg. Fisch.-Ztg. 87, 114-116 (1962). 
33 Nümann, W.: Die Auswirkungen der Eutrophierung auf den Eintritt der Reife, auf die Eizahl und Eigrösse beim Bodenseeblaufelchen (Coregonus wartmanni). Allg. Fisch.-Ztg. 88, 225-227 (1963).

34 Nümann, W.: Die Eutrophierung des Bodensees, die Zunahme der Barsche und die intensive Befischung als Ursachen für den rapiden Rückgang der Blaufelchenfänge. Allg. Fisch.-Ztg. 89, 308311 (1964).

35 Nümann, W.: Reife und Laichzeit, erblich determiniert oder von Umweltfaktoren bestimmt? Öst. Fisch. 19, 3-8 (1966).

Nümann, W.: Ungewollte und gezielte Eingriffe in die Populationsdynamik der Blaufelchen. Arch

Fisch.-Wiss. 18, 12-24 (1967)

37 Nümann, W.: The 'Blaufelchen' of Lake Constance (Coregonus wartmanni) under negative and positive influence of man. In: Lindsey, C.C., und Woods, C.S. (eds.): Biology of Coregonid fishes, $560 \mathrm{p}$. University of Manitoba Press, Winnipeg 1970.

38 Nümann, W.: Versuch einer Begründung für den Wandel in der qualitativen und quantitativen Zusammensetzung des Fischbestandes im Bodensee während der letzten 60 Jahre und eine Bewertung der Besatzmassnahmen. Schweiz. Z. Hydrol. 35, 206-238 (1974).

39 Nydegger, P.: Vergleichende limnologische Untersuchungen an sieben Schweizerseen. Beitr. Geol. Schweiz, Hydrol. 9, 1-80 (1957).

40 Nymann, O.L.: Inter- and intraspecific variations of proteins in fishes. Ann. Acad. Reg. Sci. Upsal. 9, 1-18 (1965).

41 Nymann, O.L.: Protein variations in Salmonidae. Rep. Inst. Freshw. Res. Drottningholm 47, 5-38 (1967).

42 Roth, H.: Fischereibiologische Probleme in Brienzer-, Thuner- und Bielersee. Veröffentlichung Nr.29 des Eidg. Amtes für Gewässerschutz und der Eidg. Fischereiinspektion, S. 45-79 (1972).

43 Roth, H., und Geiger, W.: Brienzersee, Thunersee, and Bielersee: effects of exploitation and eutrophication on the salmonid communities. J. Fish. Res. Bd Can. 29, 755-764 (1972).

44 Rufli, H.: Die Biologie der Coregonen im Thuner- und Bielersee. Diss. Nr. 5451, ETH Zürich, 1975.

45 Rufli, H.: Ernährung und Wachstum der Coregonen des Thuner- und Bielersees. Schweiz. Z Hydrol., in Vorbereitung.

46 Steinmann, P.: Gutachten über die Fischereiverhältnisse des Thunersees (an die Kant. Forstdirektion des Kantons Bern) (1944).

47 Steinmann, P.: Monographie der schweizerischen Koregonen. Schweiz. Z. Hydrol. 12/1-2, 13/1 (1950/51).

48 Svaerdson, G.: The Coregonid problem. I. Some general aspects of the problem. Rep. Inst. Freshw. Res. Drottningholm 29, 89-101 (1949).

49 Svaerdson, G.: Natural selection and egg number in fish. Rep. Inst. Freshw. Res. Drottningholm 29, 115-122 (1949)

50 Svaerdson, G.: The Coregonid problem. II. Morphology of two Coregonid species in different environments. Rep. Inst. Freshw. Res. Drottningholm 31, 79-125 (1950).

51 Svaerdson, G.: The Coregonid problem. III. Whitefish from the Baltic, successfully introduced into fresh waters in the north of Sweden. Rep. Inst. Freshw. Res. Drottningholm 32, 79-125 (1951).

52 Svaerdson, G.: The Coregonid problem. V. Sympatric whitefish species of the lakes Idsjön, Storsjön and Hornavan. Rep. Inst. Freshw. Res. Drottningholm 34, 141-166 (1953).

53 Svaerdson, G.: The Coregonid problem. VI: The Palearctic species and their intergrades. Rep. Inst. Freshw. Res. Drottningholm 38, 268-310 (1957).

54 Svaerdson, G.: The Coregonid problem. VII. The isolating mechanisms in sympatric species. Rep. Inst. Freshw. Res. Drottningholm 46, 95-123 (1965).

55 Svaerdson, G.: Significance of introgression in Coregonid evolution. In: Lindsey, C.C., und Woods, C.S. (eds.): Biology of Coregonid fishes, 560 p. University of Manitoba Press, Winnipeg 1970.

56 Tsuyuki, H., und Roberts, E.: Zone electrophoretic comparison of muscle myogens and blood protein of artificial hybrids of Salmonidae with their parental species. J. Fish. Res. Bd Can. 22/3, 767-773 (1965).

57 Tsuyuki, H., Uthe, J.F., Roberts, E., und Clarke, L.W.: Comparative electropherograms of Coregonus clupeaformis, Salvelinus namaycush, S. alpinus, S. malma, and S. fontinalis from the family Salmonidae. J. Fish. Res. Bd Can. 23/10, 1599-1606 (1966).

58 Utter, F.M., Ames, W.E., und Hodgins, H.O.: Transferrin polymorphism in Coho salmon (Oncorhynchus kisutch). J. Fish. Res. Bd Can. 27, 2371-2373 (1970). 
59 Utter, F.M., und Hodgins, H.O.: Phosphoglucomutase polymorphism in sockeye salmon. Comp. Biochem. Physiol. 36, 195-199 (1970).

60 Utter, F.M., Hodgins, H.O., und Johnson, A. G.: Biochemical studies of genetic differences among species and stocks of fish. Int. N. Pac. Fish. Comm. Annu. Rep. 1970, 98-101 (1972).

61 Utter, F.M., Hodgins, H.O., Allendorf, F.W., Johnson, A.G., und Mighell, J.L.: Biochemical variants in Pacific salmon and rainbow trout: their inheritance and application in population studies. In: Schröder, J.H. (ed.): Genetics and mutagenesis of fish, 356 p. Springer-Verlag, Heidelberg, New York 1973.

62 Wagler, E.: Die Coregonen in den Seen des Voralpengebietes. IX. Die Systematik der Voralpencoregonen. Int. Revue ges. Hydrobiol. 35, 345-446 (1937).

63 Ward, J.: Hierarchical grouping to optimize an objective function. J. Am. statist. Ass. 58, 236-244 (1963).

Adresse des Autors: Dr. Hans Rufli, Seenforschungslaboratorium der EAWAG/ETH, Abt. Fischereiwissenschaften, CH-6047 Kastanienbaum. 